[CONTRIBUTION FROM THE JOHN HARRISON LABORATORY OF ChEMISTRY OF THE UNIVERSity of Pennsylvania.]

\title{
ACTIVITY COEFFICIENTS AND COLLIGATIVE PROPERTIES OF ELECTROLYTES.
}

BY HERBERT S. HARNED.

Received November 9, 1921.

As a result of considerable study in recent years on the thermodynamic properties of dilute and concentrated solutions of electrolytes, the author has arrived at the following means for testing the accuracy and consistency of available activity data, for calculating other thermodynamic data therefrom, and for organizing a convenient system by means of which such properties as activity coefficients, osmotic coefficients, vapor pressures and osmotic pressures may be accurately calculated. In some instances, the method suggested by Lewis and Linhart ${ }^{1}$ for computing activity coefficients from freezing-point data is employed but, in the main, the present method differs from that employed by the above authors and by Lewis and Randall. ${ }^{2}$ Further, the results obtained, especially for solutions of potassium and sodium chlorides, differ considerably from those of Lewis and Randall. Since these differences exist, and there will be shown to be much evidence favoring the calculations here presented, it was thought to be highly desirable to state this view of the matter.

\section{Derivations of Equations.}

In a recent communication ${ }^{3}$ it was shown that the individual ion-activity coefficients (activity of the ion divided by the molal concentration) of the hydrogen, chlorine, potassium, sodium and lithium ions can be calculated by means of the empirical formula

$$
\log F_{a}=\alpha c-\beta c^{m}
$$

where $F_{a}$ is the activity coefficient of the ion, $c$ is the concentration of the electrolyte in mols per $1000 \mathrm{~g}$. of water, and $\alpha, \beta$, and $m$ are constants which differ for the different ions.

The mean activity coefficient ${ }^{2}$ of any electrolyte, $F_{a}{ }^{\prime}$, may also be expressed by an equation of similar form to (1). 'Thus

$$
\log F_{a}{ }^{\prime}=\alpha^{\prime} c-\beta^{\prime} c^{m^{\prime}}
$$

where $c$ is the molal concentration, and $\alpha^{\prime}, \beta^{\prime}$, and $m^{\prime}$ are constants. For uni-univalent electrolytes

$$
\alpha^{\prime}=\frac{\alpha_{1}+\alpha_{2}}{2} ; \beta^{\prime} c^{m^{\prime}}=\frac{1}{2}\left(\beta_{2} c^{m_{1}}+\beta_{2} c^{m_{2}}\right)
$$

where $\alpha_{1}, \beta_{1}, m_{1}$ refer to one ion, and $\alpha_{2}, \beta_{2}, m_{2}$ refer to the other. $F_{a}{ }^{\prime}$ is defined by the equation

${ }^{1}$ Lewis and Linhart, This Journal, 41, 1951 (1919).

2 Lewis and Randall, ibid., 43, 1112 (1921).

${ }^{3}$ Harned, ibid., 42, 1080 (1920). 


$$
F_{a}^{\prime}=\frac{\alpha^{ \pm}}{c\left(\begin{array}{c}
\nu^{+-\nu} \\
\nu^{+} \times \nu
\end{array}\right)^{\frac{1}{\nu}}} .
$$

In this equation $\nu^{+}$is the mol number of cations present, $\nu^{-}$is the mol number of anions present, and $\nu$ is equal to $\nu^{+}+\nu^{-}$(e. g., for $\mathrm{BaCl}_{2}$, $\nu^{+}=1, \nu^{-}=2$; for $\left.\mathrm{La}_{2}\left(\mathrm{SO}_{4}\right)_{3}, \nu^{+}=2, \nu^{-}=3\right)$ and $\alpha^{ \pm}$is the $\nu^{\text {th }}$ root of the product of the activities of all the ions. When the anion and cation have identical activity coefficients, Equations 1 and 2 are identical. In very dilute solutions, the term $\alpha^{\prime} c$ becomes negligible and Equation 2 approaches the form of the empirical equation of Lewis and Linhart, ${ }^{1}$ namely

$$
\log F_{a}{ }^{\prime}=-\beta^{\prime} c^{m^{\prime}} \text {. }
$$

In the present paper, Equation 2 will be used principally in testing the consistency of and in coördinating available activity data, and will be shown to be valid for all electrolytes studied throughout a concentration range from $0.0001 M$ to $3 M$.

By combining Duhem's equation in its exact form and Equation 1 or 2 , it becomes possible to calculate within narrow limits the vapor pressures and osmotic pressures of aqueous solutions of all electrolytes whose activity coefficients have been accurately measured. In addition, the osmotic coefficients, or, for uni-univalent electrolytes, $i-1$, where $i$ is van't Hoff's $i$, may be computed. This purely thermodynamic quantity has usually been called "the degree of dissociation." Owing to the fact that very little reliable data are to be found on these colligative properties at $25^{\circ}$, it was thought highly desirable to construct a convenient and unified method for their calculation.

(a). Vapor Pressures from the Square Root of the Activity Products of Uni-univalent Electrolytes.-Duhem's equation may be used in the following form

$$
N_{1} \mathrm{~d} F_{1}=-N_{2} \mathrm{~d} F_{2}
$$

where $N_{1}, F_{1}$ are the mol fraction and free energy of the electrolyte, and $N_{2}, F_{2}$ are the mol fraction and free energy of the water. ${ }^{4} F_{1}$ is related to the ion activities by

$$
F_{1}=R T \ln a_{1} a_{2}+\text { const. }
$$

whence

$$
\mathrm{d} F_{1}=R T \mathrm{~d} \ln a_{1} a_{2}
$$

where $a_{1}$ and $a_{2}$ are the activities of the ions of the electrolyte. By Equation 2

$$
\log F_{a}^{\prime}=\log \sqrt{F_{a} F_{b}}=\alpha^{\prime} c-\beta^{\prime} c^{m^{\prime}}
$$

or

$$
\ln \sqrt{a_{1} a_{2}}=2.3026 \alpha^{1} c-2.3026 \beta^{\prime} c^{m^{\prime}}+\ln c
$$

or

$$
\ln a_{1} a_{2}=2\left(2.3026 \alpha^{\prime} c-2.3026 \beta^{\prime} c^{m^{\prime}}+\ln c\right)
$$

'Lewis, Proc. Am. Acad. Arts Sci., 43, 259 (1907). 
Differentiation of Equation 6 gives

$$
\mathrm{d} \ln a_{1} a_{2}=2\left(2.3026 \alpha^{\prime} \mathrm{d} c+\mathrm{d} \ln c-2.3026 \beta^{\prime} m^{\prime} c^{m^{\prime}-1} \mathrm{~d} c\right) \text {. }
$$

From (4) and (5) is obtained

$$
N_{1} \mathrm{~d} \ln a_{1} a_{2}=-N_{2} \mathrm{~d} \ln a_{3}
$$

where $a_{3}$ is the activity of the water.

Substitution of $(7)$ in (8) gives

$$
\frac{2 N_{1}}{N_{2}}\left(2.3026 \alpha^{\prime} \mathrm{d} c+\mathrm{d} \ln c-2.3026 \beta^{\prime} m^{\prime} c^{m^{\prime}-1} \mathrm{~d} c\right)=-\mathrm{d} \ln a_{\mathrm{3}} .
$$

The activity of the water molecule in these solutions is related to the vapor pressure by the equation

$$
\int_{c}^{\infty} \mathrm{d} \ln a_{3}=\int_{p}^{p} \mathrm{~d} \ln p
$$

on the assumption that water vapor at these low pressures (about $20 \mathrm{~mm}$.) obeys the ideal gas law. In the above equation, $p_{0}$ is the vapor pressure of pure water and $p$ is the vapor pressure of the solution. Consequently, the integration of the left hand member of Equation 9 will be between the limits $c$ and 0 , and the right hand member between the limits $p$ and $p_{0}$. Further

$$
N_{1}=\frac{c}{55.5+c} ; N_{2}=\frac{55.5}{55.5+c}
$$

and

Hence

$$
\frac{N_{1}}{N_{2}}=\frac{c}{55.5}
$$

$$
\int_{0}^{c} \frac{2 c}{55.5}\left(2.3026 \alpha^{\prime} \mathrm{d} c+\mathrm{d} \ln c-2.3026 \beta^{\prime} m^{\prime} c^{m^{\prime}-1} \mathrm{~d} c\right)=-\int_{p_{0}}^{p} \mathrm{~d} \ln p .
$$

Integration gives

$$
\ln \frac{p_{0}}{p}=\frac{c}{55.5}\left[2+2.3026 \alpha^{\prime} c-\frac{4.6052 \beta^{\prime} m^{\prime}}{m^{\prime}+1} c^{m_{\prime}}\right]
$$

(b). The General Equation for Any Electrolyte.--In this case, we write Duhem's equation

$$
N_{1} \mathrm{~d} \ln a_{1} a_{2} \ldots a_{v}=-N_{2} \mathrm{~d} \ln a_{w}
$$

where $a_{w}$ is the activity of the water molecules. According to Equation 2

$$
\log F_{a^{\prime}}=\log \frac{\alpha^{ \pm}}{c\left(\begin{array}{c}
\nu^{+}{ }^{+-} \\
\nu^{+} \nu^{-}
\end{array}\right)^{\frac{1}{\nu}}}=\alpha^{\prime} c-\beta^{\prime} c^{m^{\prime}}
$$

or

$$
\ln a_{ \pm}=\ln \left(a_{1} a_{2} \ldots a_{\nu}\right)^{\frac{1}{\nu}}=2.3026 \alpha^{\prime} c-2.3026 \beta^{\prime} c^{m^{\prime}}+\ln c+\frac{1}{\nu} \ln \nu^{\nu^{+} \nu^{-}}
$$

or

$$
\ln \left(a_{1} a_{2} \ldots . a_{\nu}\right)=\nu\left(2.3026 \alpha^{\prime} c-2.3026 \beta^{\prime} c^{m^{\prime}}+\ln c+\frac{1}{\nu} \ln \nu^{\nu^{+}} \nu^{-}\right.
$$


Differentiation of (14) gives

$$
\mathrm{d} \ln \left(a_{1} a_{2} \ldots a_{v}\right)=\nu\left(2.3026 \alpha^{\prime} \mathrm{d} c-2.3026 \beta^{\prime} m^{\prime} c^{m^{\prime}}-1 \mathrm{~d} c+\mathrm{d} \ln c .\right.
$$

Substituting in (13), we obtain as before

$$
\int_{0}^{c} \frac{\nu c}{55.5}\left[2.3026 \alpha^{\prime} \mathrm{d} c+\mathrm{d} \ln c-2.3026 \beta^{\prime} m^{\prime} c^{m^{\prime}-1} \mathrm{~d} c\right]=-\int_{p_{0}}^{p} \mathrm{~d} \ln p
$$

which becomes on integration

$$
\ln \frac{p_{0}}{p}=\frac{c}{55.5}\left[\nu+\frac{2.3026 \nu \alpha^{\prime} c}{2}-\frac{2.3026 \nu \beta^{\prime} m^{\prime}}{m^{\prime}+1} c^{m \prime}\right]
$$

(c). Vapor Pressures of Uni-univalent Electrolytes from Individual Ion Activities.-Lewis ${ }^{4}$ has deduced the equation

$$
\frac{\left[N_{1} \mathrm{~d} \ln a_{1}+N_{2} \mathrm{~d} \ln a_{2}+\ldots\right] P_{2} \mathrm{~T}}{\mathrm{~d} N}=0 .
$$

$N_{1}, N_{2} \ldots$. are the mol fractions of the molecular and ionic species of a mixture; $a_{1}, a_{2} \ldots \ldots$, their activities. This, as was shown by Lewis, is Duhem's equation modified so as to be thermodynamically exact. In the present case, we write

$$
N_{1} \mathrm{~d} \ln a_{1}+N_{2} \mathrm{~d} \ln a_{2}+N_{3} \mathrm{~d} \ln a_{3}=0
$$

where $N_{1}, a_{1}$, and $N_{2}, a_{2}$ refer to the mol fractions and activities of the cation and anion, respectively; and $N_{3}, a_{3}$, the mol fractions and activities of the water molecule. From Equation 1

$$
\log \frac{a}{c}=\alpha c-\beta c^{m}
$$

or

$$
\text { ln } a=2.3026 \alpha c-2.3026 \beta c^{m}+\ln c
$$

where $a$ is the activity of the ion. Differentiation gives

$$
\mathrm{d} l n c=2.3026 \alpha \mathrm{d} c+\mathrm{d} \ln c-2.3026 \beta m c^{m-1} \mathrm{~d} c .
$$

Hence

$$
\left.\begin{array}{l}
\mathrm{d} l n a_{1}=2.3026 \alpha_{1} \mathrm{~d} c+\mathrm{d} \ln c-2.3026 \beta_{1} m_{1} c^{m_{1}-1} \mathrm{~d} c \\
\mathrm{~d} \ln a_{2}=2.3026 \alpha_{2} d c+\mathrm{d} \ln c-2.3026 \beta_{2} m_{2} c^{m_{2}-1} \mathrm{~d} c
\end{array}\right\}
$$

where subscripts 1 and 2 refer to the cation and anion, respectively. Further

Rearranging (18)

$$
N_{1}=N_{2}=\frac{c}{55.5+2 c} ; N_{3}=\frac{55.5}{55.5+2 c} .
$$

$$
\frac{N_{1}}{N_{3}}\left[\mathrm{~d} \ln a_{1}+\mathrm{d} \ln a_{2}\right]=-\mathrm{d} \ln a_{3} .
$$

Substitute in $18 a$ the right hand members of Equation $19 a$ for $\mathrm{d}$ ln $a_{1}$ and $\mathrm{d} \ln a_{2}$, the values obtained above for $N_{1}$ and $N_{3}$, and collect coefficients. The following is obtained, 
$\frac{c}{55.5}\left[2.3026\left(\alpha_{1}+\alpha_{2}\right) \mathrm{d} c+2 \mathrm{~d} \ln c-2.3026 \beta_{1} m_{1} c^{m_{1}-1} \mathrm{~d} c-2.3026 \beta_{2} m_{2} c^{m_{2}-1} \mathrm{~d} c\right]=-\mathrm{d} \ln a_{3}$

Hence

$\int_{1}^{c} \frac{c}{55.5}\left[2.3026\left(\alpha_{1}+\alpha_{2}\right) \mathrm{d} c+2 \mathrm{~d} l n c-2.3026 \beta_{1} m_{1} c^{m_{1}-1} \mathrm{~d} c-2.3026 \beta_{2} m_{2} c^{m_{2}-1} \mathrm{~d} c\right]=-\int_{p_{0}}^{p} \mathrm{~d} \ln p$

Integration of this last equation gives

$$
\ln \frac{p_{0}}{p}=\frac{c}{55.5}\left[2+\frac{2.3026\left(\alpha_{1}+\alpha_{2}\right) c}{2}-\frac{2.3026 \beta_{1} m_{1}}{m_{1}+1} c^{m_{1}}-\frac{2.3026 \beta_{2} m_{2}}{m_{2}+1} c^{m_{2}}\right]
$$

(d). Osmotic Pressures and Osmotic Coefficients.- Let the expressions within the brackets of the right hand members of Equations 12, 16, and 20 equal [i]. Then

$$
\ln \frac{p_{0}}{p}=\frac{c}{55.5}[i]
$$

The osmotic pressure can be calculated from the vapor pressure (neglecting the small term due to the compressibility of the solvent) by the formula

$$
\pi=\frac{R T}{V_{0}} \ln \frac{p_{0}}{p}=\frac{R T}{V_{0}} \frac{c}{55.5}[i]
$$

where $\pi$ is the osmotic pressirre, $V_{0}$ the molal volume of the water at temperature $T$ and $R$ is the gas constant. On inspection, it is seen that $[i]$ is van't Hoff's $[i]$, and that $\left(\frac{i-1}{v-1}\right)$ is equal to the osmotic coefficient $f_{0},{ }^{5}$ or the classic degree of dissociation. At $25^{\circ}$ Equation 22 reduces to

$$
\pi \text { (atms.) }=24.42 c[i]
$$

on substituting 298.1 for $T, 0.08207$ for $R$, and 0.018053 for $V_{0}$.

\section{The Activity Coefficients of Potassium Chloride Solutions.}

In order to determine the parameters of Equation 2 and thus the activity coefficients of potassium chloride solutions, a method has been adopted which combines the method suggested by Lewis and Linhart ${ }^{1}$ for computing activity coefficients from freezing-point data with the method employed by the author ${ }^{3}$ for computing the same at higher concentrations from electromotive-force measurements. Lewis and Linhart found that, in dilute solutions of all electrolytes, the following empirical relationship holds, namely,

$$
\log \left(\nu \lambda-\frac{\theta}{c}\right)=\alpha_{1}^{\prime} \log c+\log B_{1}^{\prime}
$$

where $\nu$ is as before the number of different ions produced in the solution by the dissociating substance, $\lambda$ the molal freezing-point lowering of water $\left(1.858^{\circ} \mathrm{C}\right.$ ),$\theta$ the freezing-point lowering, $c$ the concentration, and $\alpha_{1}^{\prime}$ and $\beta_{1}{ }^{\prime}$ are constants $\left(\alpha_{1}{ }^{\prime}, \beta_{1}{ }^{\prime}\right.$ are the constants $\alpha_{1}, \beta_{1}$ in Lewis and Linhart's

5 Bjerrum, Z. anorg. Chem., 109, 275 (1920). 
paper). By plotting $\log \left(\nu \lambda-\frac{\theta}{c}\right)$ against $\log c$, straight lines are obtained from which the values of $\alpha_{1}{ }^{\prime}$ and $\beta_{1}{ }^{\prime}$ can be evaluated. By combining this equation with the thermodynamic equation

$$
\int \mathrm{d} \ln F_{a}^{\prime}=\frac{1}{\nu \lambda} \int \frac{\mathrm{d} \theta}{c}
$$

which relates the activity coefficient with the freezing-point lowering in dilute solutions, and integrating between the proper limits, they obtained

$$
\log F_{a}^{\prime}=-\frac{\beta_{1}{ }^{\prime}\left(\alpha_{1}{ }^{\prime}+1\right) c^{\alpha_{1}}}{2.3026 \nu \lambda \alpha_{1}{ }^{\prime}}
$$

Since for a given electrolyte $\alpha_{1}{ }^{\prime}, \beta_{1}{ }^{\prime}, \nu$, and $\lambda$ are constants, this equation is equivalent to (3) where

$$
\beta^{\prime}=\frac{\beta_{1}{ }^{\prime}\left(\alpha_{1}{ }^{\prime}+1\right)}{2.3026 \nu \lambda \alpha_{1}{ }^{\prime}} ; \text { and } m^{\prime}=\alpha_{1}^{\prime} .
$$

Thus, by plotting freezing-point data in accordance with Equation 24, the parameters $\beta^{\prime}$ and $m^{\prime}$ of Equation 2 may be evaluated. Employing this method with solutions of potassium chloride, Lewis and Linhart obtained $\alpha_{1}{ }^{\prime}=0.535 ; \beta_{1}{ }^{\prime}=1.223$ which according to Equations 27 give the values $\beta^{\prime}=0.401 ; m^{\prime}=0.535$. The author obtained $\beta^{\prime}=0.318$ and $m^{\prime}=$ 0.397 , calculated from electromotive-force data and based on the value $F_{a(0.1)}^{\prime}=0.754$ at $0.1 M$ concentration of Noyes and MacInnes; ${ }^{6}$ Lewis and

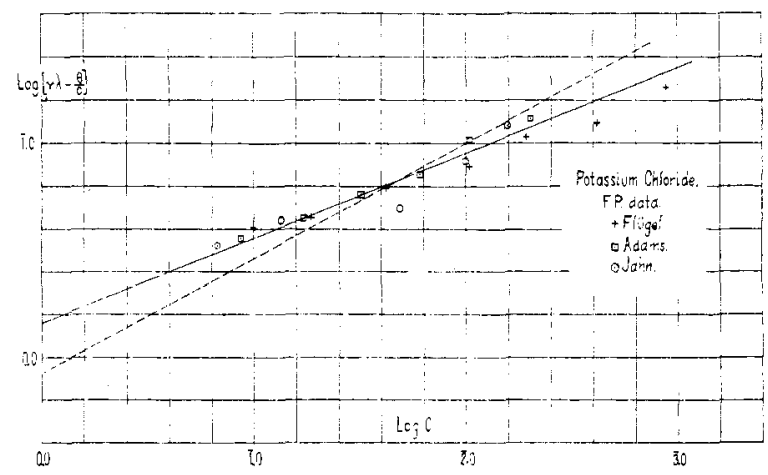

Fig. 1.

Randall ${ }^{2}$ obtained $F^{\prime} a_{(0.1)}=0.798$. The large differences between the values of these constants as determined by Lewis and Linhart and by the author make a recalculation imperative. Instead of basing the calculation entirely upon the freezing-point data of Adams, ${ }^{7}$ it was thought preferable to include also the data of Flügel ${ }^{8}$ and of Jahn. ${ }^{9}$ In Fig. 1, the values of

- Noyes and Macinnes, This Journal, 42, 239 (1920).

7 Adams, ibid., 37, 494 (1915).

S Flügel, Z, physik. Chem., 79, 585 (1912).

- Jahn, ibid., 59, 35 (1907). 
$\log \left({ }_{\nu \lambda}-\frac{\theta}{c}\right)$ are plotted against $\log c$. It can be seen that these points group along a straight line between $\log c=\overline{1} .00$ and $\log c=\overline{2} .00$, and lie near a straight line at lower concentrations. At concentrations above $0.1 \mathrm{M}$, Equation 24 is no longer valid. The dotted line represents the straight corresponding to values of $\alpha_{1}{ }^{\prime}$ and $\beta_{1}{ }^{\prime}$ obtained by Lewis and Linhart. Their plot passes through some points obtained from Adams' data, and does not represent the line drawn through the points of all the data. The representative curve gives $\alpha_{1}{ }^{\prime}=m^{\prime}=0.394$ and $\beta_{1}{ }^{\prime}=0.692$ from which, by $(27), \beta^{\prime}=0.286$. Employing these values in Equation $3 F^{\prime}{ }_{(0,1)}=$ 0.766. But Equation 3, is not applicable up to concentrations as high as 0.1 $M$, and consequently, in order to obtain a more exact value for $F^{\prime}{ }_{(0.1)}$, Equation 2 is employed, assigning to $\alpha^{\prime}$ the value 0.070 , and to $\beta^{\prime}$ and $m^{\prime}$ the values obtained above. In the previous article, $\alpha^{\prime}$ was assigned the value 0.080 . The corrected value for $F^{\prime}{ }_{(\mathbf{r}, 1)}$ is 0.779 . Using this value, the activity coefficients have been computed at higher concentrations from the electromotiveforce data of Noyes and MacInnes ${ }^{6}$ and Harned ${ }^{10}$ and compiled in Table I.

TABLE I

Activity Coefficients from Electromotive Force Data

$\begin{array}{cccc}c & F_{a}^{\prime} \text { (obs.) } & & F_{a}^{\prime} \text { (calc.) } \\ \text { Noyes and MacInnes } & \text { Harned } & \begin{array}{c}\alpha^{\prime}=0.070 \\ \beta^{\prime}=0.292 \\ m^{\prime}=0.396\end{array} \\ 0.03 & 0.851 & \ldots & 0.858 \\ 0.05 & 0.816 & \ldots .779 & 0.821 \\ 0.10 & 0.779 & 0.776 \\ 0.20 & 0.723 & \ldots & 0.724 \\ 0.30 & 0.695 & 0.687 & 0.693 \\ 0.50 & 0.659 & 0.660 & 0.652 \\ 0.70 & 0.639 & \ldots & 0.625 \\ 0.75 & \ldots & 0.619 & 0.620 \\ 1.00 & 0.613 & 0.598 & 0.600 \\ 1.50 & \ldots & 0.575 & 0.578 \\ 2.00 & \ldots & 0.566 & 0.570 \\ 2.50 & \ldots & 0.568 & 0.567 \\ 3.00 & \ldots & 0.575 & 0.574\end{array}$

The observed values are lower in all cases than those given in Table XIV in Lewis and Randall's paper. The parameters $\alpha^{\prime}, \beta^{\prime}, m^{\prime}$ can be derived from these data by the method previously employed by the author. Thus

$$
\log F_{a}^{\prime}=\alpha^{\prime} c-\beta^{\prime} c^{m^{\prime}} \text {. }
$$

By transposition is obtained

$$
\left[-\frac{\log F_{a}{ }^{\prime}}{c}+\alpha^{\prime}\right]=B^{\prime} c^{m-1}
$$

${ }^{10}$ Harned, This Journal, 38, 1986 (1916). 
and taking the logarithms

$$
\log \left[-\frac{\log F_{a^{\prime}}}{c}+\alpha^{\prime}\right]=\log \beta^{\prime}+\left(m^{\prime}-1\right) \log c
$$

Thus, the plot of $\log \left[-\frac{\log F_{a}^{\prime}}{c}+\alpha^{\prime}\right]$ against $\log c$ should be a straight line. The plot was made by assigning different values to $\alpha^{\prime}$ until a straight line was obtained. $m^{\prime}$ can readily be determined from the slope, and $\beta^{\prime}$ from the value of $\log \beta^{\prime}$ at $\log c=0$ or $c=1$. Fig. 2 gives this plot from which are obtained $\alpha^{\prime}=0.070 ; \beta^{\prime}=0.292$; and $m^{\prime}=0.396$. From freezingpoint measurements, $\beta^{\prime}=0.286$, and $m^{\prime}=0.394$. The agreement is excellent. Using the data of Lewis and Randall ${ }^{2}$ (Table XIV), and plotting

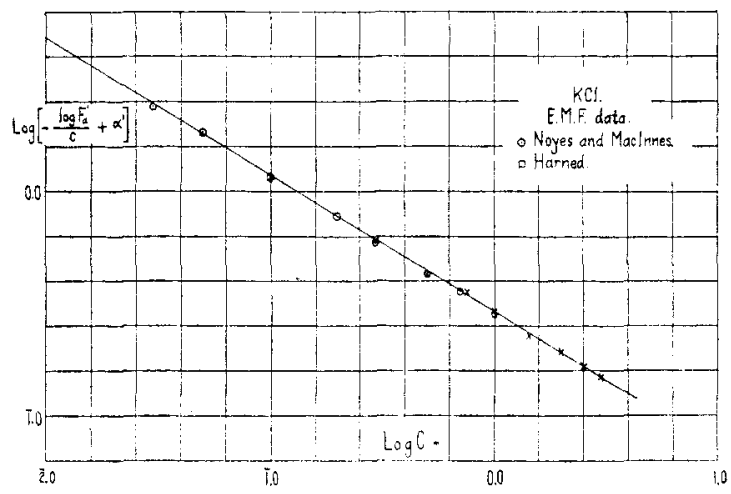

Fig. 2.

in the same way as above, a straight line is obtained which gives $\alpha^{\prime}=0.134$, $\beta^{\prime}=0.334$, and $m^{\prime}=0.474$. In what follows, it will be shown that the lower values obtained by the author are more exact. The only lack of concordance between the above calculation and observed data occurs in the electromotive-force measurements of Noyes and MacInnes below $0.01 M$. Lewis and Randall suggest that electromotive-force measurements at these low concentrations are not reliable owing to side reactions. At present, there are not sufficient data from different sources to decide this question definitely. Having determined the values of $\alpha^{\prime}, \beta^{\prime}$ and $m^{\prime}$, we are now in a position to compare the results with other carefully determined data.

By computing the values of the expression inside the bracket of the right hand member of Equation 12 and subtracting 1 from these values, the osmotic coefficients at the different concentrations are obtained. Equation 12 becomes on substituting the values for $\alpha^{\prime}, \beta^{\prime}$ and $m^{\prime}$ obtained above

whence

$$
\ln \frac{p_{0}}{p}=\frac{c}{55.5}\left[2+0.1612 c-0.3815 c^{0.396}\right]
$$

$$
f_{0}=1+0.1612 c-0.3815 c^{0.396} \text {. }
$$


Table II, Col. 2 contains the values of $f_{0}$ at the concentrations denoted in Col. 1 calculated by $(12 b)$. Col. 3 contains the values of $f_{0}$ using the values of $\alpha^{\prime}, \beta^{\prime}$ and $m^{\prime}$ from Lewis and Randall's data. 'The last two columns of this table contain values of $f_{0}$ at $0^{\circ}$ and $20^{\circ}$ calculated by means of Equation 21 from values of $\ln \frac{p_{0}}{p}$. The latter clata were obtained from Table II in a recent paper by Lovelace, Frazer, and Sease. ${ }^{11}$ The values at $0^{\circ}$ were calculated by them from freezing-point lowerings using an exact thermodynamic formula given by Washburn, ${ }^{12}$ the freezing-point data of Roloff ${ }^{13}$ and the compilation of Noyes and Falk. ${ }^{14}$ The $20^{\circ}$ values were obtained from their own vapor-pressure measurements.

TABLE II

OSMOTIC COEFFICIENTS

(1)

(2)

(3)

(4)

Conc.

E.m.f.

f. $f$ and Lewis and

F. p. data

f. p. Randall

$0^{\circ}$

$25^{\circ} \quad 25^{\circ}$

$\alpha^{\prime}=0.070 \quad \alpha^{\prime}=0.134$

$\beta^{\prime}=0.292 \quad \beta^{\prime}=0.334$

$m^{\prime}=0.396 \quad m^{\prime}=0.474$

0.001

0.976

0.010

0.940

$\cdots$

0.050

0.892

0.951

0.100

0.863

0.903

0.877

0.812

0.821

0.791

0.820

0.781

0.826

0.780

0.814

0.792

0.900

0.820

0.973

0.855

1.055

0.894

1.145

$\ldots$
$\ldots$
0.891
0.857
0.810
0.794
0.762
0.748
0.764
0.763
0.765
0.805

$(5)$
V.p.

V. p.

(Obs.) (Obs.)

Eq. 28 L.F. and

(Mannite) S.

3.000

0.984

Lewis and Randall's values are higher than any of the other series of results. The above data reveal that the plot of the osmotic coefficients against the concentrations will have a minimum. The plot of the molecular vapor-pressure lowerings against the concentrations will also have a minimum at the same concentration.

The empirical equation which relates the osmotic coefficient to the concentration is

$$
f_{0}=1+\alpha^{\prime \prime} c-\beta^{\prime \prime} c^{m^{\prime}}
$$

\footnotetext{
${ }^{11}$ Lovelace, Frazer and Sease, This Journal, 43, 102 (1921).

${ }_{12}$ Washburn, "Principles of Physical Chemistry," McGraw Hill Book Co., 1915, p. 169.

1 Roloff, Z. physik. Chem., 18, 578 (1895).

14 Noyes and Falk, Jil Tus JourNaL, 32, 1020 (1910).
} 
where $\alpha^{\prime \prime}$ and $\beta^{\prime \prime}$ are the numerical coefficients in Equation $12 b$ and $m^{\prime}$ is the same as in the previous equations. Differentiating (12c) with respect to $c$ and equating the right hand member of the resulting equation to (), the following is obtained as a condition for a minimum

$$
\alpha^{\prime \prime}=\beta^{\prime \prime} m^{\prime} c^{m^{\prime}-1} \text {. }
$$

Employing this latter equation, it was found that the minimum of the results in Table II, Col. 2 occurs at $c$ equals 0.898 . The minimum from Lewis and Randall's data occurs at $c$ equals 0.525 . Lovelace, Frazer and Sease obtained a minimum in the molecular vapor-pressure-lowering-concentration plot $\left(20^{\circ}\right)$ in the neighborhood of $1 \mathrm{M}$ concentration.

The last column in Table II contains a series of osmotic coefficients of potassium chloride solutions at $20^{\circ}$, computed from measurements of vaporpressure lowerings obtained by Lovelace, Frazer and Sease. ${ }^{11}$ The agreement between these results and the values obtained by the author and given in Col. 2 is good but not entirely satisfactory. At concentrations from $0.05 M$ to $0.5 M$ their results are lower than those obtained by the author, from $0.5 M$ to $2 M$ their values are slightly higher; and from $2 M$ up their results are lower. The $5^{\circ}$ difference in temperature will cause some difference which would depend on the sign of the reversible molal heat of dilution by water. Since the latter quantity is negative for potassium chloride solutions, the osmotic coefficients should be higher at $25^{\circ}$ than at $20^{\circ}$. In the results at concentrations from 0.5 to $2 M$ the slight error may be in either the vapor-pressure or electromotive-force measurements. The results from the data of Lovelace $e$ al. at $0.05 \mathrm{M}$ and $0.1 \mathrm{M}$ are unquestionably low. This is verified by comparison with the values obtained by the above authors from measurements of both potassium chloride and mannite solutions, where any small error due to their apparatus would be partially eliminated. Since mannite solutions obey Raoult's law within narrow limits, $f_{0}$ may be calculated by the equation

$$
\frac{\left(p_{0}-p\right)_{\mathrm{KOl}}}{\left(p_{0}-p\right)_{\text {manite }}}=1+f_{0}
$$

where $\left(p_{0}-p\right)$ is the vapor-pressure lowering of the substance denoted by the subscript. In Table II, Col. 5 are given the values of $f_{0}$ obtained by employing Equation 28. The agreement between these results and those in Col. 2 is good.

In order to carry the investigation somewhat further and to relate in a general way the osmotic coefficients from the freezing-point, vapor-pressure, and electromotive-force data with the reversible molal heat of dilution by water, it was thought advisable to compute the parameters of Equation 2 from the freezing-point and vapor-pressure data (Table II, Cols. 4 and 5) and then to make an approximate calculation of the reversible molal heat of dilution by water. Transposing and taking the log of both members of Equation $12 c$, we obtain 


$$
\log \left[1-f_{0}+\alpha^{\prime \prime} c\right]=\log \beta^{\prime \prime}+m^{\prime} \log c
$$

where

$$
\alpha^{\prime \prime}=2.3026 \alpha^{\prime} ; \text { and } \beta^{\prime \prime}=\frac{4.065 \beta^{\prime} m^{\prime}}{m^{\prime}+1} .
$$

By plotting the $\log \left[1-f_{0}+\alpha^{\prime \prime} c\right]$ against the $\log c$, a straight line should be obtained if $\alpha^{\prime \prime}$ be assigned the correct value. From this plot $\beta^{\prime \prime}$ and $m^{\prime}$ can readily be evaluated. In Fig. 3 , the plots of the values obtained from

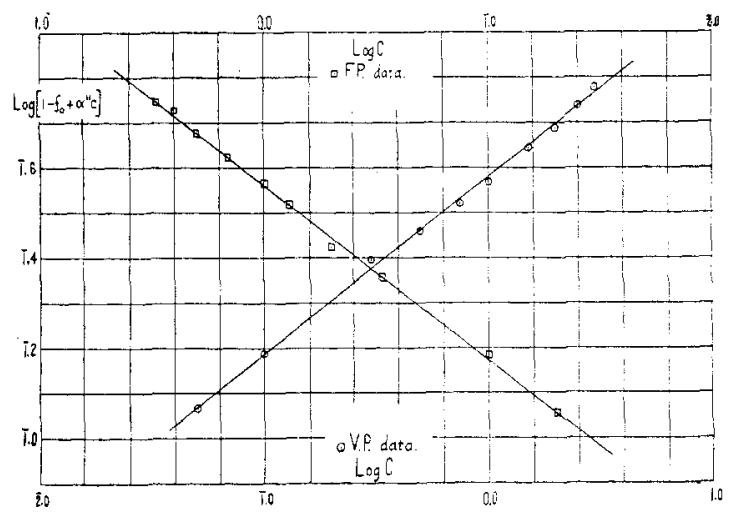

Fig. 3.

the freezing-point data ('lable II, Col. 4) and vapor-pressure measurements (Table II, Col.5) are shown. 'The values in parenthesis were omitted. In Table III are given the values of $\alpha^{\prime}, \beta^{\prime}$, and $m^{\prime}$ obtained from these

\begin{tabular}{|c|c|c|}
\hline \multicolumn{3}{|c|}{ TABIE III } \\
\hline li. p. $\left(0^{\circ}\right)$ & V.p. $\left(20^{\circ}\right)$ & li.t11.f. $\left(25^{\circ}\right)$ \\
\hline 0.052 & 0.068 & 0.070 \\
\hline 0.277 & 0.292 & 0.292 \\
\hline$m^{\prime} \ldots \ldots \ldots 0.3 \times 7$ & 0.30 .5 & 0.397 \\
\hline
\end{tabular}
plots, and from the previous calculation.

'The values from the freezing-point data conform very nearly to the straight line. The vapor-pressure data points do not fall so close to the straight. Lovelace, Frazer and Sease report that in the neighborhood of 0.4 to $0.8 \mathrm{M}$, the molecular vapor-pressure-lowering-concentration plot shows an irregularity not due to experimental error. Such an irregularity has never yet been found in electromotive-force measurements. For potassium chloride solutions, it would be observed between 1.5 and $2.0 \mathrm{M}$ salt concentrations.

Calculation of the osmotic coefficients using the values of $\alpha^{\prime}, \beta^{\prime}$, and $m^{\prime}$ in Table III will give values which increase with rise in-temperature. From the fundamental thermodynamic equation 


$$
\frac{\mathrm{d}\left(\mathrm{d} \ln \frac{p_{0}}{p}\right)}{\mathrm{d} T}=\frac{\Delta \bar{H}}{R T^{2}}
$$

and Equation 21, it follows that if the osmotic coefficient increases with the temperature, then $\frac{\mathrm{d}\left(\ln \frac{p_{0}}{p}\right)}{\mathrm{d} T}$ is positive, and $\Delta \bar{H}$, the partial molal heat content function of dilution is also positive. Hence, the reversible molal heat of dilution will be negative. This is in agreement with the data of Stearn and Smith. ${ }^{15}$ Further, the temperature coefficient $\frac{d\left(\ln \frac{p_{0}}{p}\right)}{d T}$ decreases as the temperature rises so that in the neighborhood of from $20^{\circ}$ to $25^{\circ}$, it is quite small. From the values of $\alpha^{\prime}, \beta^{\prime}$, and $m^{\prime}$ in Table IIT, [i] was calculated and thence $\left.\ln \left(\frac{p_{0}}{p}\right)\right]_{20}$ and $\left.\ln \left(\frac{p_{0}}{p}\right)\right]_{26}$ were obtained by Equation 21. The temperature coefficient at $25^{\circ}$ was taken as the difference $\left.\left.\left[\ln \left(\frac{p_{0}}{p}\right)\right]_{25}-\ln \left(\frac{p_{0}}{p}\right)\right]_{20}\right]$ divided by 5 . This mean coefficient is slightly higher than the true coefficient at $25^{\circ}$ and, consequently, when $\Delta H$ is calculated from this value, it should be slightly greater than the observed result. The final results of this calculation are given in T'able IV.

TABLE IV IinAt, RESULTS

$\begin{array}{cllll}c & \left.\ln \frac{p_{0}}{p}\right]_{25} & \left.\ln \frac{p_{0}}{p}\right]_{35} & \frac{\left.\left.\left(\ln \frac{p_{0}}{p}\right]_{25}-\ln \frac{p_{0}}{p}\right]_{20}\right)}{5} & \begin{array}{c}(-\Delta \bar{H}) \\ \text { Reversible } \\ \text { molal heat of }\end{array} \\ 1.000 & 0.03207 & 0.03198 & 0.000018 & <-3.2 \\ 2.000 & 0.06559 & 0.06523 & 0.000072 & <-12.7 \\ 3.000 & 0.1024 & 0.1013 & 0.00022 & <-38.8\end{array}$

Steart and Smith obtained $(-30.0)$ at $3.16 \mathrm{M} ;(-10.8)$ at $1.6 \mathrm{M}$; and $(-3.2)$ at $0.8 M$. This is excellent agreement, and a splendid verification of the correctness of the original activity coefficients.

\section{The Activity Coefficients of Sodium Chloride and Hydrochloric Acid Solutions.}

The same method as used in the calculation of the activity coefficients of potassium chloride is employed with sodium chloride solutions, and need orly be described briefly. The $\log \left(\nu \lambda-\frac{\theta}{c}\right)$ was plotted against $\log c$ ${ }^{15}$ Stearn and Smith, This JoURNaI, 42, 18 (1920). 
using the freezing-point data of Harkins and Roberts, ${ }^{16}$ and Flügel. ${ }^{8}$ The plot is shown in Fig. 4 from which $\alpha_{1}{ }^{\prime}=m^{\prime}=0.413$; and $\beta_{1}{ }^{\prime}=0.720$ which by Equation 27 gives $\beta^{\prime}=0.288$. Using these values in Equation 3, we obtain $F_{a_{(0,1)}}=0.774$. Assigning the value of 0.100 to $\alpha^{\prime}$ and employing Equation 2, $F_{a_{(0.1)}}=0.792$. This value will be accepted. The

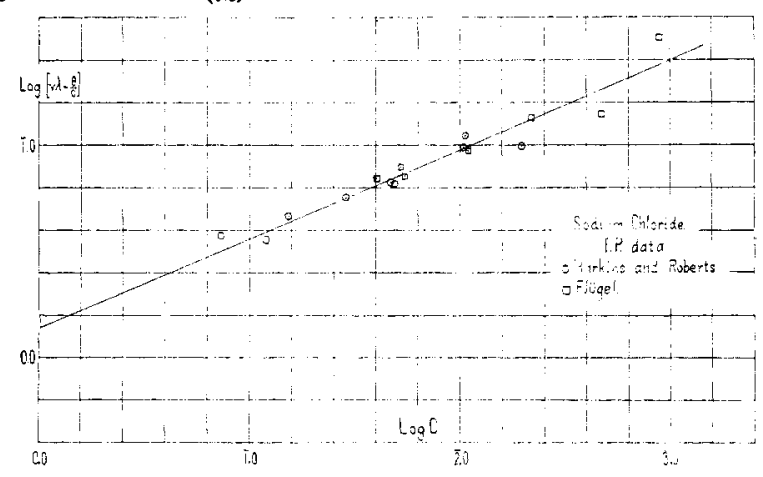

Fig. 4.

activity coefficients at concentrations above $0.1 \mathrm{M}$ can now be calculated from the electromotive-force data of Allmand and Polack ${ }^{17}$ and of Harned. ${ }^{18}$ These are compiled in Table $\mathrm{V}$.

TABLE V

\begin{tabular}{|c|c|c|c|}
\hline$c$ & $\begin{array}{c}F_{a}{ }^{\prime} \text { (obs.) } \\
\text { A. and P. }\end{array}$ & $\begin{array}{c}F_{a}^{\prime} \text { (obs.) } \\
\text { Harned }\end{array}$ & $\begin{array}{c}F_{a}^{\prime} \text { (calc.) } \\
\alpha^{\prime}=0.100 \\
\beta^{\prime}=0.286 \\
m^{\prime}=0.407\end{array}$ \\
\hline 0.1003 & 0.791 & 0.791 & 0.792 \\
\hline 0.301 & $\ldots$ & 0.691 & 0.715 \\
\hline 0.5034 & 0.686 & 0.671 & 0.674 \\
\hline 1.022 & 0.653 & 0.664 & 0.651 \\
\hline 2.000 & & 0.693 & 0.679 \\
\hline 3.196 & 0.735 & $\ldots$ & 0.726 \\
\hline 6.12 (sat.) & 1.019 & . & 1.033 \\
\hline
\end{tabular}

Using the sedata, $\log \left[\frac{-\log F_{a}^{\prime}}{c}+\alpha^{\prime}\right]$ has been plotted against $\log c$ (Fig. 5). A straight line is obtained when $\alpha^{\prime}=0.100$. From this plot $\beta^{\prime}=0.286$ and $m^{\prime}=0.407$ which is again excellent agreement with the values of the same constants from freezing-point data. The values of $\alpha^{\prime}, \beta^{\prime}$, and $m^{\prime}$ differ to a considerable extent from those computed by the same method from the results in Table XIV of Lewis and Randall's paper. From their data are obtained $\alpha^{\prime}=0.140 ; \beta^{\prime}=0.335$; and $m^{\prime}=0.480$.

${ }^{16}$ Harkins and Roberts, This JouRnaL, 38, 2676 (1916).

${ }_{17}$ Allmand and Polack, J. Chem. Soc., 115, 1020 (1919).

18 Harned, This JouRNAL, 40, 1462 (1918). 
Fig. 5 also contains the plot of $\log \left[\frac{-\log F_{a}^{\prime}}{c}+\alpha^{\prime}\right]$ against $\log c$ for hydrochloric acid solutions. The activity data were taken from the electromotive-force data of Ellis, ${ }^{19}$ and Noyes and Ellis. ${ }^{20}$ From this plot, $\alpha^{\prime}=0.200 ; \beta^{\prime}=0.286$; and $m^{\prime}=0.434$.

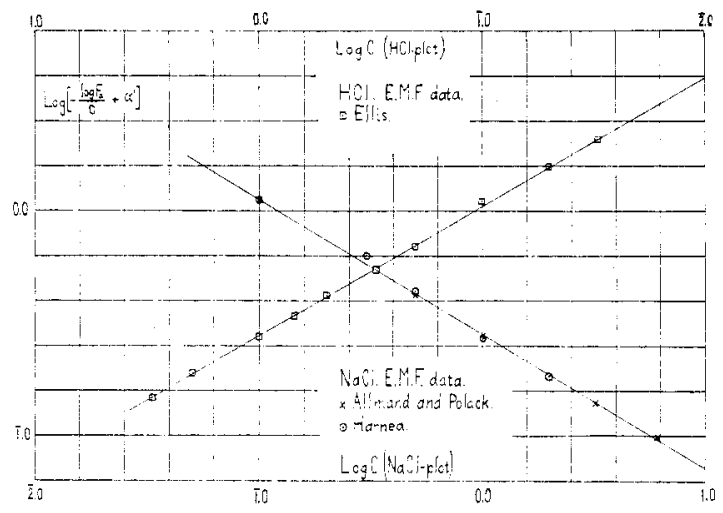

Fig. 5.

Table VI contains a summary of the values for the parameters $\alpha^{\prime}$, $\beta^{\prime}$, and $m^{\prime}$ obtained in this paper and the values of $\alpha, \beta$, and $m$ computed therefrom for the individual ions on the assumption that $F_{a_{\mathbf{K}}}=F_{a_{\mathrm{Cl}}}$ and that the ions act independently. ${ }^{3}$ These values for the independent ionactivity coefficients are to be regarded as an approximation and are subject to change as a result of further accumulation of evidence.

\begin{tabular}{|c|c|c|c|}
\hline \multicolumn{4}{|c|}{$\begin{array}{l}\text { TABLE VI } \\
\text { SUMMARY }\end{array}$} \\
\hline & $\mathrm{KCl}$ & $\mathrm{NaCl}$ & $\mathrm{HCl}$ \\
\hline $\begin{array}{l}\alpha^{\prime} \ldots \ldots \ldots \ldots \ldots \\
\beta^{\prime} \ldots \ldots \ldots \ldots \ldots \\
m^{\prime} \ldots \ldots \ldots \ldots \ldots\end{array}$ & $\begin{array}{l}0.070 \\
0.292 \\
0.396\end{array}$ & $\begin{array}{l}0.100 \\
0.286 \\
0.407\end{array}$ & $\begin{array}{l}0.200 \\
0.288 \\
0.434\end{array}$ \\
\hline$\alpha$ & $\begin{array}{c}\mathrm{K}^{+}=\mathrm{Cl}^{-} \\
0.070\end{array}$ & $\begin{array}{c}\mathrm{Na}^{+} \\
0.130\end{array}$ & $\begin{array}{c}\mathrm{H}^{+} \\
0.330\end{array}$ \\
\hline$\ldots \ldots \ldots \ldots$ & 0.292 & 0.280 & 0.284 \\
\hline$m \ldots \ldots \ldots \ldots$ & 0.396 & 0.417 & 0.471 \\
\hline
\end{tabular}

'These values stubstituted in Equations 12, 20 and 22 will give numerical equations by means of which the vapor pressures and osmotic pressures of these solutions at $25^{\circ}$ may be accurately calculated.

\section{Calculation of the Colligative Properties of Other Electrolytes.}

The values of $\alpha^{\prime}, \beta^{\prime}$, and $m^{\prime}$ for the different electrolytes in Table XIV in I,ewis and Randall's paper have been obtained by the same method as

19 Ellis, This Journal, 38, 737(1916).

${ }^{20}$ Noyes and Ellis, ibid., 39, 2532(1917). 
above and compiled in Table VII. In the second column are given the ranges of concentration over which the values of the constants may be regarded as valid. The values of these parameters with the possible exception of lithium chloride and hydrochloric acid are not as accurate as the values in Table VI and are subject at any time to revision.

TABLE VII

Values Calculated hrom Lewis and Randall's Paper

\begin{tabular}{|c|c|c|c|c|}
\hline Electrolyte & $\begin{array}{l}\text { Range of } \\
\text { validity }\end{array}$ & $\alpha^{\prime}$ & $\beta^{\prime}$ & $m^{\prime}$ \\
\hline $\mathrm{LiCl}\left(25^{\circ}\right)$ & $0.01-3$ & 0.200 & 0.323 & 0.463 \\
\hline $\mathrm{KNO}_{3} \ldots \ldots$ & $0.01-0.1$ & . & 0.497 & 0.558 \\
\hline $\mathrm{AgNO}_{3}$. & $0.01-5$ & & 0.406 & 0.470 \\
\hline $\mathrm{KIO}_{3}, \mathrm{NaIO}_{3}$ & $0.01-0.1$ & $\ldots$ & 0.469 & 0.468 \\
\hline $\mathrm{BaCl}_{2} \ldots \ldots \ldots \ldots$ & $0.01-0.1$ & 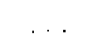 & 0.643 & 0.327 \\
\hline $\mathrm{CdCl}_{2}\left(25^{\circ}\right) \ldots \ldots \ldots$ & $0.01-0.1$ & $\ldots$ & 1.901 & 0.441 \\
\hline $\mathrm{K}_{2} \mathrm{SO}_{4} \ldots \ldots \ldots \ldots$ & $0.01-0.1$ & $\ldots$ & 0.873 & 0.363 \\
\hline $\mathrm{H}_{2} \mathrm{SO}_{4} \ldots \ldots$ & $0.01-3$ & 0.470 & 1.365 & 0.402 \\
\hline $\left.\mathrm{L} a(\mathrm{NO})_{3}\right)_{3} \ldots$ & $0.01-0.2$ & $\ldots$ & 0.933 & 0.286 \\
\hline $\mathrm{MgSO}_{4} \ldots \ldots$ & $0.01-0.1$ & $\ldots$ & 1.496 & 0.290 \\
\hline $\mathrm{CdSO}_{4}$ & $0.01-0.2$ & $\ldots$ & 1.611 & 0.306 \\
\hline$\ldots \ldots \ldots$ & $0.01-0.5$ & & 1.496 & 0.290 \\
\hline
\end{tabular}

By using Equation 12 or 16 , accurate values of the vapor pressures of these solutions may be computed; and by using Equation 22 the osmotic pressures may be obtained.

\section{The Limit of Validity of Equation 2.}

In all cases yet studied, Equation 2 has been found to hold within the present experimental error throughout the entire concentration range of available data with the exception of sulfuric and hydrochloric acids. Lewis

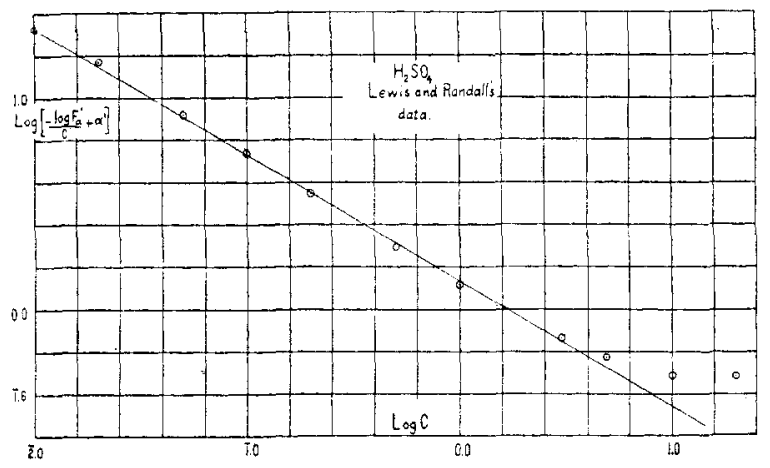

Fig. 6.

and Randall have obtained the activity coefficients of sulfuric acid from 0.01 to 20 molal. The plot of $\log \left[\frac{\log F_{a}^{\prime}}{c}+\alpha^{\prime}\right]$ against $\log c$ is shown 
in Fig. 6 whence can be seen that the curve departs from a straight line above $3 M$ concentration.

\section{Summary.}

$\therefore$ On the basis of an empirical equation

$$
\log F_{a^{\prime}}=\alpha^{\prime} c-\beta^{\prime} c^{m \prime}
$$

which relates the activity coefficient of an electrolyte with the molal concentration at a given temperature, and Duhem's equation, equations are derived for calculating the vapor pressures of solutions at this temperature.

2. A rigorous test was made of the validity of Equation 2. It was found to hold for all electrolytes throughout the concentration ranges where available data were examined with the exception of sulfuric and hydrochloric acids. In these cases it holds up to $3 M$ concentration.

3. A very careful and exhaustive study of the activity coefficients of potassium chloride solutions has been made.

4. A careful but less exhaustive study of the activity coefficients of sodium chloride solutions and hydrochloric acid solutions has been made.

5. Values of the parameters of Equation 2 obtained for the above 3 and numerous other electrolytes have been collected and tabulated, from which the vapor pressures and osmotic pressures of their solutions may be calculated.

It is thought that the equation and method here employed, although empirical and an approximation will prove useful in testing the consistency and accuracy of activity data and also will prove of some service in organizing the colligative data of concentrated solutions.

Phit,adelphia, PenNsylvania.

[Contribution from the Bureau of Standards, U. S. Derartment of Commerce.]

\section{A SIMPLE THEORY OF THE NEPHELOMETER. ${ }^{1}$}

BY P. V. WELLS.

Received November 9, 1921.

In recent years several instruments have been developed using as a measuring criterion the light diffusely reflected and transmitted by suspensions, and variously called turbidimeters, tyndallmeters and nephelometers. At present there is on the market the Kober nephelometer ${ }^{2}$ (a development of the modification by Richards and Wells of the Dubosq colorimeter) which measures the ratio of the depths of two suspensions of equal brightness either by diffuse reflection or transmission.

These instruments are used to obtain information about the suspension

1 Published by permission of the Director of the Bureau of Standards.

${ }^{2}$ Made by Klett Mfg. Co., 202 E. 46th St., New York City. 